\title{
CCAAT/enhancer-binding protein $\beta$ regulates interleukin-6-induced transmembrane and ubiquitin-like domain containing 1 gene expression in hepatocytes
}

\author{
MENGGANG LIU, TAO YUAN, HONGMING LIU and PING CHEN \\ Department of Hepatobiliary Surgery, Daping Hospital, The Third Military Medical University, Chongqing 400042, P.R. China
}

Received October 29, 2013; Accepted May 21, 2014

DOI: $10.3892 / \mathrm{mmr} .2014 .2457$

\begin{abstract}
Transmembrane and ubiquitin-like domain containing 1 (Tmub1) protein has a negative effect on liver regeneration; however, the mechanisms of Tmub1 expression regulation are currently unknown. CCAAT/enhancer-binding protein $\beta(\mathrm{C} / \mathrm{EBP} \beta)$ is a key transcription factor associated with interleukin-6 (IL-6) in liver regeneration. The present study aimed to investigate the involvement of $\mathrm{C} / \mathrm{EBP} \beta$ in the IL-6-induced upregulation of Tmub1. A series of Tmub1 luciferase reporter vectors were constructed to detect the effects of IL-6 on the transcriptional activity of Tmub1. Small interfering RNA and overexpression vectors were employed to either inhibit or enhance the expression of $\mathrm{C} / \mathrm{EBP} \beta$, respectively, to determine the effect of $\mathrm{C} / \mathrm{EBP} \beta$ on Tmub1 expression in liver cells. Additionally, chromatin immunoprecipitation experiments were performed to determine whether $\mathrm{C} / \mathrm{EBP} \beta$ bound to the Tmub1 gene, and the sequences associated with the regulation of Tmub1 expression by $\mathrm{C} / \mathrm{EBP} \beta$ were subsequently determined. Several potential binding sites of $\mathrm{C} / \mathrm{EBP} \beta$ were identified in the 5'-upstream region of the Tmubl gene. $\mathrm{C} / \mathrm{EBP} \beta$ expression was positively correlated with Tmub1 gene expression in rat liver cells. Furthermore, it was demonstrated that $\mathrm{C} / \mathrm{EBP} \beta$ bound to 5'-upstream sequences of the Tmub1 gene to enhance the promoter activity. The present study demonstrated that $\mathrm{C} / \mathrm{EBP} \beta$ is a key transcription factor, which can positively regulate the expression of Tmub1 during liver cell proliferation through a possible association with IL-6.
\end{abstract}

Correspondence to: Professor Ping Chen, Department of Hepatobiliary Surgery, Daping Hospital, The Third Military Medical University, 10 Changjiangzhilu Daping, Chongqing 400042, P.R. China

E-mail: chenping10701@hotmail.com

Abbreviations: $\mathrm{C} / \mathrm{EBP} \beta, \mathrm{CCAAT} / \mathrm{enhancer}^{-b i n d i n g}$ protein $\beta$; Tmub1, transmembrane and ubiquitin-like domain containing 1; STAT3, signal transducer and activator of transcription-3; IL-6, interleukin-6

Key words: C/EBP $\beta$, Tmub1, IL-6, liver regeneration

\section{Introduction}

Liver regeneration is an adaptive defense mechanism to protect against external injury. Regeneration occurs through a highly orchestrated and complex hepatic hyperplasia process, involving numerous growth factor signaling cascades, cytokines, extracellular matrix remodeling and an interactive network balance between stimulatory and inhibitory factors for hepatocyte proliferation (1). These processes are precisely controlled by transcription factors to promote and terminate liver tissue regeneration through three phases: i) Initiation, ii) proliferation and iii) termination (2). The activities of transcription factors allow their target genes to be transcribed rapidly. The transcriptional targets encode various proteins that are essential to induce hepatocytes to leave the quiescent state $\left(\mathrm{G}_{0}\right)$ and enter the cell cycle (2). Following acute liver injury or hepatectomy, the loss of hepatic parenchyma induces a high expression of tumor necrosis factor $\alpha$, lymphotoxin, interleukin-1 (IL-1) and IL-6 (3-5). These cytokines and growth factors activate transcription factors such as nuclear factor $\kappa$-light-chain-enhancer of activated $B$ cells $(\mathrm{NF}-\kappa \mathrm{B})$, signal transducer and activator of transcription-3 (STAT3), activator protein 1 (AP-1) and CCAAT/enhancer-binding protein $\beta(\mathrm{C} / \mathrm{EBP} \beta)$ in the remaining liver cells to initiate proliferation and DNA synthesis. This process can be dynamically adjusted through the increase of proliferative inhibitory factors to maintain liver size during tissue restoration $(6,7)$.

IL-6 is a critical initiation factor for liver regeneration. During the initiation phase of liver regeneration, IL-6 is secreted by activated Kupffer cells, enabling hepatocytes to undergo mitosis by directly regulating proliferation-related gene expression, such as hepatocyte growth factor expression, and protecting hepatocytes from apoptosis (8). This process is primarily mediated by the mitotic transcription factor STAT3, through the canonical pathway, to regulate the liver regeneration process (8). Numerous binding sites for other transcription factors are usually found adjacent to the STAT3 binding site and these factors cooperate to regulate gene expression. It has been shown that STAT3 functions together with $\mathrm{C} / \mathrm{EBP} \beta$, $\mathrm{AP}-1$ and $\mathrm{NF}-\kappa \mathrm{B}$ to regulate the expression of a large network of liver regeneration of genes $(9,10)$.

The C/EBP family of transcription factors is characterized by a basic region/leucine zipper domain, which is involved in the regulation of liver-specific gene expression, with an impor- 
tant role in liver development and liver cell function $(11,12)$. $\mathrm{C} / \mathrm{EBP} \beta$ is an important member of the $\mathrm{C} / \mathrm{EBP}$ family as it has been shown that $\mathrm{C} / \mathrm{EBP} \beta$ deficiency can result in liver regeneration failure, indicating an essential role for $\mathrm{C} / \mathrm{EBP} \beta$ during regeneration $(8,13) . \mathrm{C} / \mathrm{EBP} \beta$ (also known as NF-IL-6) is a downstream target of IL-6 $(14,15)$ and is activated through the Ras/mitogen-activated protein kinase pathway to induce gene expression during the acute phase of liver regeneration (16). A previous study also found that $\mathrm{C} / \mathrm{EBP} \beta$ promoter sequences contain an acute phase response element, and DNA binding experiments have demonstrated that STAT3 binds to this element to activate transcription of $\mathrm{C} / \mathrm{EBP} \beta$ (17).

Although less comprehensively studied, pathways leading to the inhibition of liver regeneration are considered to be equally important as those initiating the process. Transmembrane and ubiquitin-like domain containing 1 (Tmub1) is a candidate molecule for involvement in the termination of liver regeneration; Tmub1 was first identified by Della Fazia et al (18), who showed that its expression was markedly elevated during liver regeneration. Additionally, overexpression of Tmub1 has been shown to play a role in the inhibition of cell proliferation (18). Our previous study found that short-hairpin RNA knockdown of Tmubl expression increased liver cell proliferation, indicating that Tmub1 may play a negative role in liver regeneration (19). However, a regulatory function for Tmub1 expression is not clear. The previous results demonstrated that IL- 6 could enhance the expression of Tmub1 in rat liver cells (19). C/EBP $\beta$ expression was significantly increased $2 \mathrm{~h}$ after partial hepatectomy (20), whilst the peak of Tmub1 expression appeared later, $48 \mathrm{~h}$ after surgery (18). This indicates that $\mathrm{C} / \mathrm{EBP} \beta$ may participate in the regulation of Tmubl gene expression. Through bioinformatics analysis in the present study, it was found that the Tmub1 promoter sequence contained $\mathrm{C} / \mathrm{EBP} \beta$ and STAT3 binding sites. The aims of the present study were three-fold: i) To characterize the association between $\mathrm{C} / \mathrm{EBP} \beta$ and Tmub1 gene expression; ii) to determine whether $\mathrm{C} / \mathrm{EBP} \beta$ could bind the Tmub1 promoter by chromatin immunoprecipitation (ChIP) assay; and iii) to analyze the effects of $\mathrm{C} / \mathrm{EBP} \beta$ on Tmub1 promoter activity to predict the potential binding sites of $\mathrm{C} / \mathrm{EBP} \beta$ in the Tmubl gene.

\section{Materials and methods}

Cell culture. Normal BRL-3A rat hepatocytes (Cell Bank of Academia Sinica, Shanghai, China) were cultured in high-glucose Dulbecco's Modified Eagle's medium with 10\% fetal bovine serum (Invitrogen Life Technologies, Carlsbad, $\mathrm{CA}, \mathrm{USA})$ at $37^{\circ} \mathrm{C}$ with $5 \% \mathrm{CO}_{2}$.

Bioinformatics analysis of promoter sequences of Tmubl gene. According to available information on the rat Tmub1 gene (GenBank NC_005103.2; http://www.ncbi.nlm.nih. gov/genbank/), online software (http://www-bimas.cit. nih.gov/molbio/proscan/; version 1.7) was used to predict the potential promoter regions of Tmub1, using 53 as the cut-off value for prediction. Online software (http://cbrc. $\mathrm{jp} / \mathrm{research} / \mathrm{db} / \mathrm{TFSEARCH} . \mathrm{html}$ ) was used to predict the potential transcription factor binding sites in the upstream 5 ' end sequence of the Tmub1 gene. The maximum score was 100.0 and the minimum score was 85.0.
Small interfering RNA (siRNA) knockdown. Three targeted siRNAs and one scramble control siRNA were synthesized by RiboBio Co. (Guangzhou, China) against rat C/EBP $\beta$. siRNA was transfected using Lipofectamine ${ }^{\mathrm{TM}} 2000$ according to the manufacturer's instructions (Invitrogen Life Technologies). Western blot analysis was performed to determine the optimal siRNA sequence and concentration for the inhibition of $\mathrm{C} / \mathrm{EBP} \beta$ at $72 \mathrm{~h}$ post-transfection.

Construction of the rat $C / E B P \beta$ overexpression vector and cell transfection. mRNA was isolated from BRL-3A cells and reverse transcribed into cDNA (Takara, Tokyo, Japan). The sense and antisense primers for $\mathrm{C} / \mathrm{EBP} \beta$ amplification were as follows: 5'-ATATGGATCCATGCACCGCCTGCTGGCCT-3' and 5'-ATAACTCGAGCTAGCAGTGACCCGCCGAGG-3', respectively. The full-length amplified $\mathrm{C} / \mathrm{EBP} \beta \mathrm{cDNA}$ was inserted into a pCMVTag2B vector (Kang Chen Bio-tech Inc., Shanghai, China) and the sequence was verified by polymerase chain reaction (PCR) and sequencing. $\mathrm{C} / \mathrm{EBP} \beta$ expression vectors were transfected using a Lipofectamine 2000 kit (Invitrogen Life Technologies) and cells were harvested for testing at $72 \mathrm{~h}$ post-transfection.

Quantitative PCR ( $q$ PCR) analysis. RNA was extracted from cells using TRIzol ${ }^{\circledR}$ reagent (Takara) and used for reverse transcription using a High Capacity RNA-to-cDNA kit (Takara) according to the manufacturer's instructions. PCR reactions were prepared using the Brilliant SYBR Green qPCR Master Mix kit (Takara) and amplified using a Bio-Rad CFX96 thermocycler (Bio-Rad, Hercules, CA, USA). The sense and antisense primers were as follows: Tmub1, 5'-GTAGGCGATGAGGTGACTGT-3' and 5'-GCTGTGCTG GTGTTGTGG-3' (fragment length, 136 bp); C/EBP $\beta, 5$ '-CAC CGGGTTTCGGGACTTG-3' and 5'-CCCGCAGGA ACATCTTTAAGTG-3' (fragment length, 128 bp); $\beta$-actin internal reference, 5'-ACGTTGACATCCGTAAAGAC-3' and 5'-GAAGGTGGACAGTGAGGC-3' (fragment length, 200 bp).

Western blot analysis. Cells were collected and washed with phosphate-buffered saline, lysed in radioimmunoprecipitation assay buffer on ice and disrupted by sonication. Protein samples were separated by SDS-PAGE and transferred to a membrane. The membrane was blocked with $5 \%$ non-fat dry milk buffer for $1 \mathrm{~h}$ and immunoblotted with Tmub1 and anti-C/EBP $\beta$ primary and goat anti-rat secondary antibodies (Santa Cruz Biotechnology Inc., Santa Cruz, CA, USA). Protein bands were imaged by an Odyssey Two Infrared Imaging system (LI-COR, Lincoln, NE, USA) and analyzed by Quantity One software (Bio-Rad).

ChIP assay. A ChIP assay was performed using the EZ-ChIP kit (Millipore, Billerica, MA, USA) according to the manufacturer's instructions. The following antibodies were used: Anti-RNA polymerase as a positive control, normal rat immunoglobulin $\mathrm{G}$ as a negative control (Santa Cruz Biotechnology Inc.) and anti-C/EBP $\beta$ antibody in the experimental groups. Primer 5 software (Premier Biosoft, Palo Alto, CA, USA) was used to design the following primers for Tmub1 amplification: Forward primer, 5'-TCTGCAAGCATAAGGACCTC-3' and reverse primer, 5'-ACTGAGCTAAATCCCCATCC-3', 
Table I. Forward primer sequences for the construction of Tmub1 reporter gene vectors.

\begin{tabular}{llr}
\hline Primer & \multicolumn{1}{c}{ Sequence $\left(5^{\prime}{ }^{\prime}-3^{\prime}\right)$} & Fragment length $(\mathrm{bp})$ \\
\hline T1 & ATAAACGCGTTACATATAGAAACCCCATCCTGAAA & 2058 \\
T2 & ATAAACGCGTGCATATAAAACATATATATTTAA & 1784 \\
T3 & TTAAACGCGTGCCCAGGGCAATATTTGAT & 1687 \\
T4 & ATAAACGCGTGTAGAGTATTTTAATTCAGACT & 1609 \\
T5 & AAAAACGCGTCATGCAGATATAATATACAAACC & 1442 \\
T6 & ATATACGCGTCAACCTTAGCAGAGGGAGCAG & 1317 \\
T7 & ATAAACGCGTCTCTAGGTGGCCAAAGGTAGT & 926 \\
T8 & ATATACGCGTCGTTTCTTCTTTCCAACTGA & 729 \\
T9 & ATAAACGCGTCGAGGGCAATTCACGCCTCCT & 517 \\
T10 & ATAAACGCGTAAGCTGGCGGCGGAAATAGAGT & 323 \\
T11 & ATAAACGCGTCTACCGGTACCGGGAACATCT & 202 \\
T12 & ATATACGCGTTCCCGGGCCGCGGCAGCA & 107 \\
\hline
\end{tabular}

Tmub1, transmembrane and ubiquitin-like domain containing 1; bp, base pairs.

(fragment length, $208 \mathrm{bp}$ ). PCR reactions were carried out to validate whether $\mathrm{C} / \mathrm{EBP} \beta$ interacted with the sequences within the promoter region of Tmub1.

Construction of Tmubl reporter gene vectors with varying promoter lengths. Genomic DNA from the BRL-3A cell line was used as a template to amplify 12 fragments in the 5 ' upstream sequence $(-2,007$ to +50$)$ of the Tmubl gene. The forward primer is shown in Table I and the reverse primer was as follows: 5'-ATAACTCGAGACCCAGCTCCACCA GCTCCATT-3'. The promoter fragments were subcloned into digested pGL3-Basic vectors (KangChen Bio-tech Inc, Shanghai, China), and positive clones were identified by PCR and sequencing.

Detection of luciferase reporter gene activity. BRL-3A cells were cultured in 48-well plates to $70-80 \%$ confluency prior to co-transfection with Tmub1 promoter-luciferase reporter vectors and $\mathrm{C} / \mathrm{EBP} \beta$ expression plasmid or a negative control. Transfection was performed using Lipofectamine 2000 according to the manufacturer's instructions. After $48 \mathrm{~h}$ in culture (depending on the experiment, $15 \mathrm{ng} / \mathrm{ml}$ IL-6 was added $12 \mathrm{~h}$ prior to the luciferase activity assay), cells were harvested and assayed using a Dual Luciferase Assay kit (Promega Corp., Madison, WI, USA) according to the manufacturer's instructions.

Statistical analysis. All data are presented as the mean \pm standard deviation. Statistical analysis was performed with Sigma Plot 10.0 software (Systat Software Inc., San Jose, CA, USA). Differences between individual groups were analyzed by least-significant difference test. A value of $\mathrm{P}<0.05$ was considered a statistically significant difference.

\section{Results}

Bioinformatics analysis of the 5' upstream sequence of Tmubl. Based on the information from GenBank, it was found that
Table II. Distribution of potential transcription factor binding sites upstream of the Tmub1 gene.

\begin{tabular}{lc}
\hline Transcription factor & Potential binding sites (bp) \\
\hline C/EBP $\beta$ & -1751 to -1738 \\
C/EBP $\beta$ & -1702 to -1689 \\
AP-1 & -1455 to -1445 \\
C/EBP $\beta$ & -1344 to -1331 \\
C/EBP & -794 to -781 \\
AP-1 & -727 to -717 \\
AP-1 & -664 to -654 \\
CREB & -411 to -404 \\
CREB & -240 to -137 \\
C/EBP & -240 to -228 \\
STAT & -145 to -137
\end{tabular}

STAT, signal transducer and activator of transcription; AP-1, activator

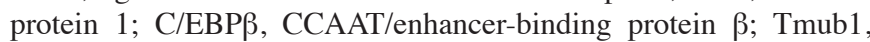
transmembrane and ubiquitin-like domain containing 1 ; bp, base pairs; CREB, cAMP response element-binding protein.

the transcription start site of the rat Tmubl gene was located 1,092 bp upstream of the translational initiation codon (ATG). GC-rich regions were found at $+50 \mathrm{bp}$ upstream of the transcription start point, which could be part of the first intron sequences and binding sites for the promoter or enhancer of the Tmub1 gene. Online software analysis revealed that the promoter binding sites were more likely to be located within the -322 to -122 bp region (score 69.64). TFSEARCH software analysis revealed that within the $-2,008$ to +50 bp region, there were multiple potential binding sites for transcription factors associated with liver regeneration and IL-6 signaling pathways, including five C/EBP sites (5), three AP-1 sites (3), a STAT site (1) and two cAMP response element-binding protein sites (2) (Table II). 
IL-6 upregulation of Tmubl expression may correlate with $C / E B P \beta, S T A T 3$ and AP-1 transcription factors. A series of Tmub1 luciferase reporter vectors, known as T1-T12, were constructed (Fig. 1A) and transfected into BRL-3A cells to determine the Tmub1 promoter activity. In the absence of stimulating factors, all of the sequence fragments showed significant transcriptional activity $(\mathrm{P}<0.05)$. With the exception of the T4 and T6 promoter fragments, which maintained promoter activity, the transcriptional activities of the other promoter fragments were gradually decreased by deleting the Tmub1 promoter region sequences. When the $-1,392$ to $-1,268$ bp sequence was deleted, the transcriptional activity of the $\mathrm{T} 6$ fragment was markedly elevated, indicating that there was a potential repressive element of Tmub1 expression within the deleted sequence (Fig. 1B). Following the addition of IL-6, the luciferase reporter activity in most reporter vectors was significantly increased $(\mathrm{P}<0.05)$, with the exception of the T6 group. The luciferase activities among the groups were also substantially variable (Fig. 1B). IL-6 could enhance transcriptional activity of the Tmubl promoter, and deleting different sequences resulted in differential effects on the IL-6-induced activities: Deletion of the $-2,008$ to $-1,735$ (T2), $-1,734$ to 1,638 (T3), $-1,559$ to 1,393 (T5), $-1,392$ to 1,268 (T6), -273 to 153 (T11) and -152 to -58 bp (T12) sequences had a more marked effect. These sequences contained potential binding sites for C/EBP $\beta$, STAT3 and AP-1. The majority of these sites were $\mathrm{C} / \mathrm{EBP} \beta$ (or C/EBP) binding sites, which were located at 2,008 to $-1,735,-1,734$ to $-1,638,-1,392$ to $-1,268$ and -273 to -153 bp.

C/EBP $\beta$ expression is positively correlated with Tmubl expression in rat liver cells. Upon siRNA knockdown of C/EBP $\beta$ expression, Tmub1 mRNA and protein levels significantly decreased in liver cells. Conversely, upon overexpression of $\mathrm{C} / \mathrm{EBP} \beta$, Tmub1 mRNA and protein levels were increased (Fig. 2). These results suggest that $\mathrm{C} / \mathrm{EBP} \beta$ levels are positively correlated with Tmub1 expression and that $\mathrm{C} / \mathrm{EBP} \beta$ may be involved in the regulation of Tmub1 expression during liver cell proliferation.

$C / E B P \beta$ can bind to 5' upstream sequences of the Tmubl gene. ChIP assay was used to determine whether C/EBP $\beta$ could bind to Tmub1 and directly regulate its expression in rat liver cells. From bioinformatics analysis, a fragment sequence in the Tmub1 promoter region was selected that contained one $\mathrm{C} / \mathrm{EBP} \beta$ binding site (-1,344 to $-1,331 \mathrm{bp})$ and this was used as a target sequence in the experiment (Fig. 3). The PCR results demonstrated that the DNA sequence binding to $\mathrm{C} / \mathrm{EBP} \beta$ contained the promoter sequence of the Tmub1 gene.

$C / E B P \beta$ can enhance the promoter activity of Tmub1. To refine the potential binding sites of C/EBP $\beta$ in Tmubl, luciferase reporter gene vectors were used to observe the effects of $\mathrm{C} / \mathrm{EBP} \beta$ on the promoter activity of Tmub1 upon enhancing C/EBP $\beta$ expression (Fig. 4). With the exception of the T6 fragment, the transcriptional activities of the other 11 constructs were significantly increased $(\mathrm{P}<0.05)$. The most prominent effects on the transcriptional activity of the promoter were obtained by deleting the $-2,008$ to $-1,735$ (T2), -273 to -153 (T11) and -152 to -58 bp (T12) sequences, indicating that the binding sites of $\mathrm{C} / \mathrm{EBP} \beta$ may be located within these three
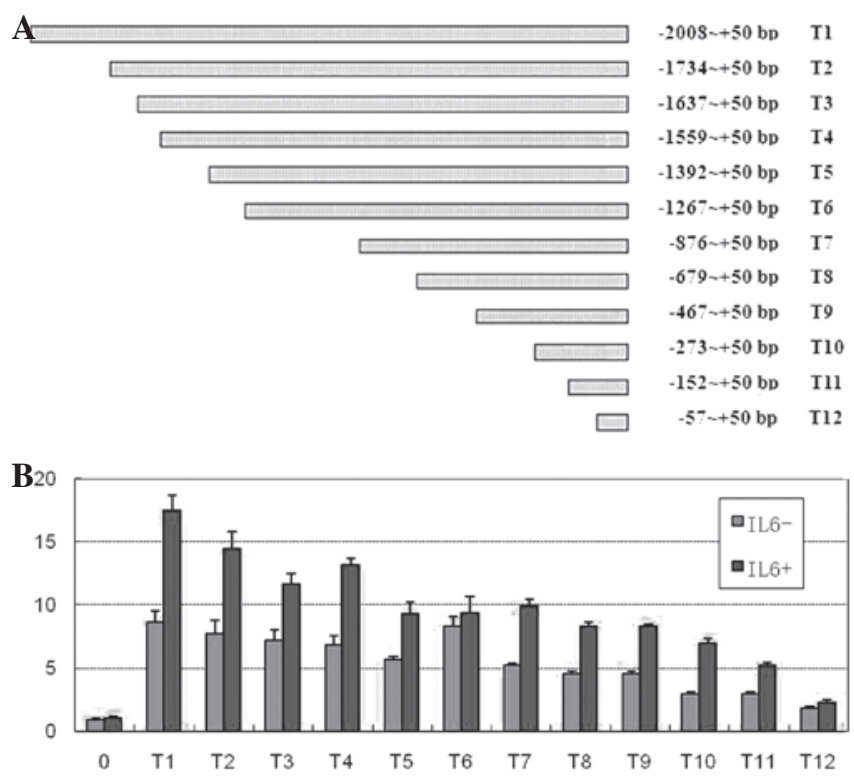

Figure 1. (A) Schematic of Tmub1 luciferase reporter vectors. A series of deletion constructs of the Tmub1 promoter (T1-T12) were generated and subcloned into pGL3-Basic vectors. (B) Activities of Tmub1 luciferase reporter vectors induced by IL-6 in BRL-3A cells. The empty pGL3-Basic vector (T0 control group) and a series of Tmub1 luciferase reporter vectors (T1-T12, experimental groups) were transfected into BRL-3A cells for $48 \mathrm{~h}$, and a luciferase activity assay was performed with or without IL-6 addition. Luciferase activities of the experimental groups were significantly higher than those of the control group $(\mathrm{P}<0.05)$. Error bars represent the mean \pm standard deviation. bp, base pairs; IL6, interleukin-6; Tmub1, transmembrane and ubiquitin-like domain containing 1.

fragment sequences. Although the potential repressive element of Tmub1 expression could lie within the $-1,392$ to $-1,268 \mathrm{bp}$ sequence, the luciferase activity of the $\mathrm{T} 5$ reporter vector was also enhanced following C/EBP $\beta$ overexpression, and the deletion of this fragment sequence did not significantly alter luciferase activity. This suggests that the $\mathrm{C} / \mathrm{EBP} \beta$ binding site may lie within this fragment sequence and the transcriptional enhancement caused by $\mathrm{C} / \mathrm{EBP} \beta$ interacting with the binding site can offset the inhibitory role of the repressive element.

\section{Discussion}

Our previous study demonstrated that Tmub1 plays a negative regulatory role in the hepatocyte proliferation process; however, its expression can be upregulated by the positive regulators of proliferation, such as IL-6 (19). There are little data regarding the inhibitory factors affecting proliferation during hepatic regeneration. Thus, clarifying the regulatory mechanism of Tmubl expression is important in understanding the association between a proliferative promoting factor and a proliferative inhibitory factor in the hepatocyte regeneration process and the mechanism of hepatocyte homeostasis.

The results of the present study indicated that IL- 6 may regulate Tmub1 expression during liver cell proliferation. The expression of IL-6 target genes ultimately depends on the activities of its downstream transcription factors. In order to explore the regulatory mechanism of Tmub1 expression, the transcription factors involved in the regulation of Tmub1 expression by IL- 6 were first identified. The effects of IL- 6 on 
A

a

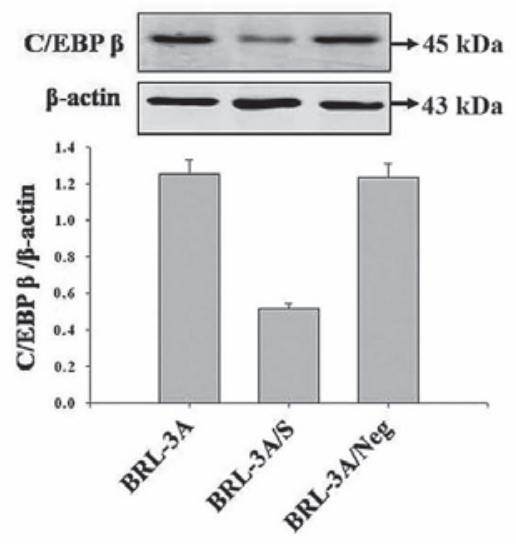

c
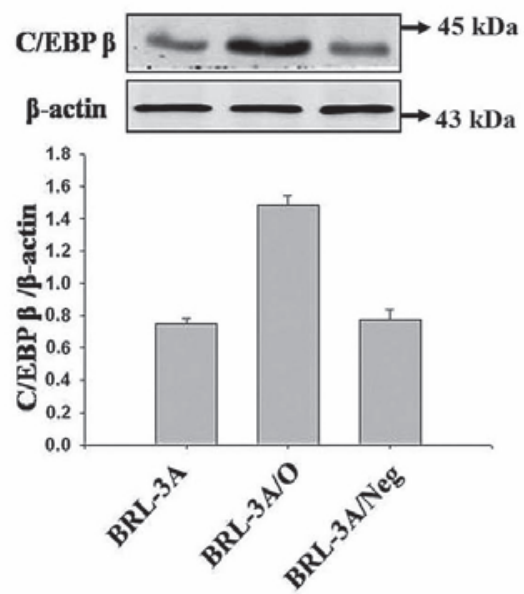

b
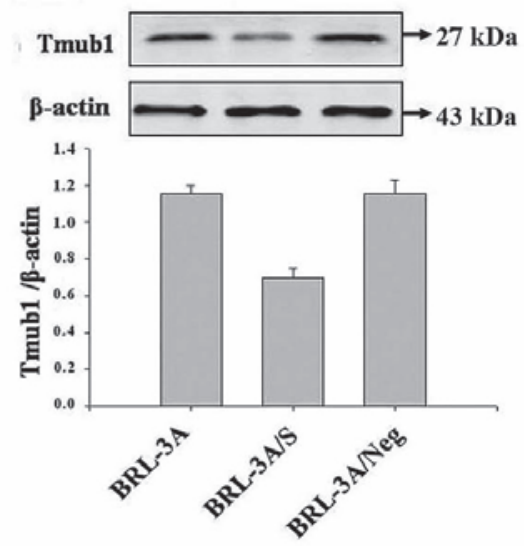

d
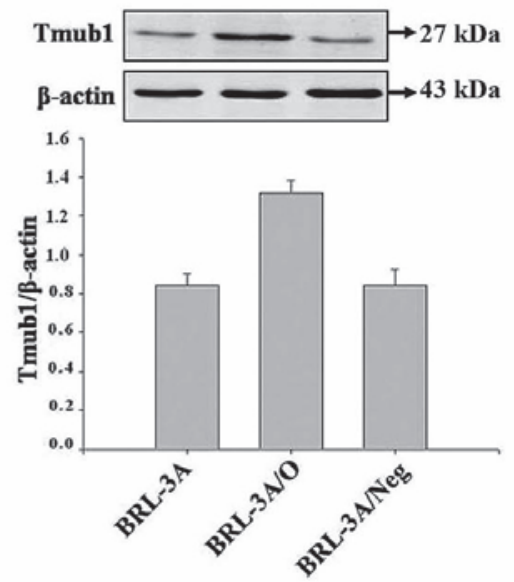

B

a

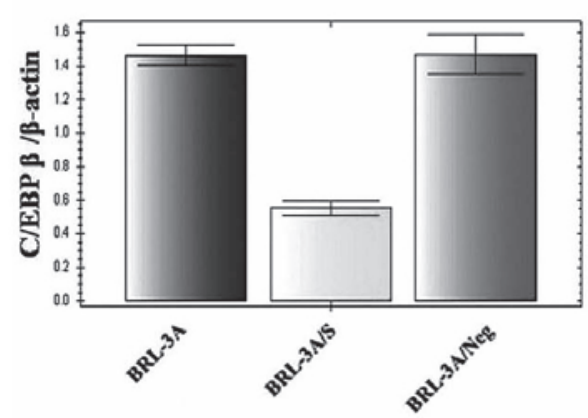

c

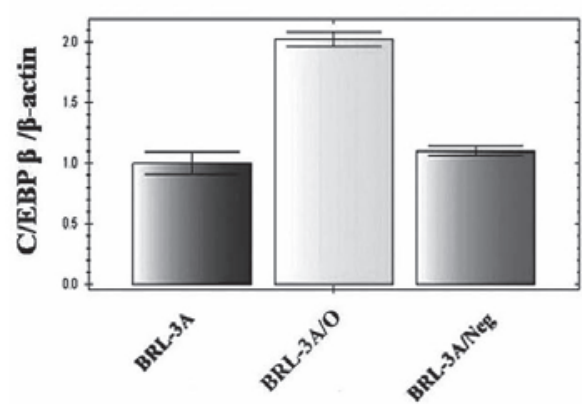

b

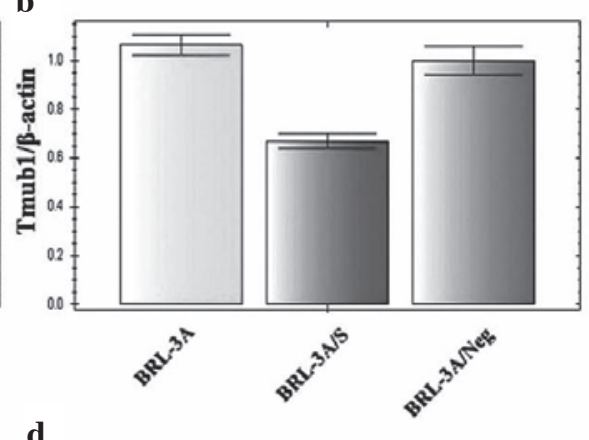

d

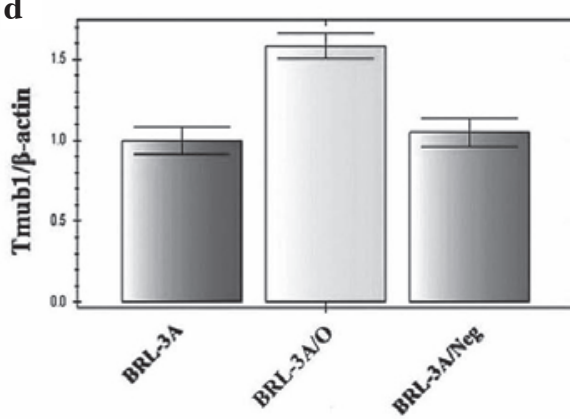

Figure 2. Expression of $\mathrm{C} / \mathrm{EBP} \beta$ and Tmubl is positively correlated in rat liver cells, as demonstrated by (A) western blotting and (B) quantitative polymerase chain reaction of whole cell lysates. (a) BRL-3A cells were transfected with target siRNA for C/EBP $\beta$ (BRL-3A/S) or negative control siRNA (BRL-3A/Neg) or underwent a mock transfection without siRNA (BRL-3A). (b) BRL-3A cells were transfected with target siRNA for Tmub1 (BRL-3A/S) or negative control siRNA (BRL-3A/Neg) or underwent a mock transfection without siRNA (BRL-3A).(c) BRL-3A cells were transfected with expression vector for C/EBP $\beta$ (BRL-3A/O) or negative control vector (BRL-3A/Neg) or underwent a mock transfection without vector (BRL-3A). (d) BRL-3A cells were transfected with expression vector for Tmub1 (BRL-3A/O) or negative control vector (BRL-3A/Neg) or underwent a mock transfection without vector (BRL-3A). The expression of (a) $\mathrm{C} / \mathrm{EBP} \beta$ and (b) Tmub1 in the BRL-3A/S group was signifcantly lower than that in the control groups $(\mathrm{P}<0.05)$. The expression of (c) $\mathrm{C} / \mathrm{EBP} \beta$ and (d) Tmub1 in the BRL-3A/O group was signifcantly higher than that in the control groups $(\mathrm{P}<0.05)$. Expression of $\mathrm{C} / \mathrm{EBP} \beta$ and Tmub1 was not signifcantly different in the BRL-3A and BRL-3A/Neg groups $(\mathrm{P}>0.05)$. Error bars represent the mean \pm standard deviation. C/EBP $\beta$, CCAAT/enhancer-binding protein $\beta$; Tmub1, transmembrane and ubiquitin-like domain containing 1; siRNA, small interfering RNA. 


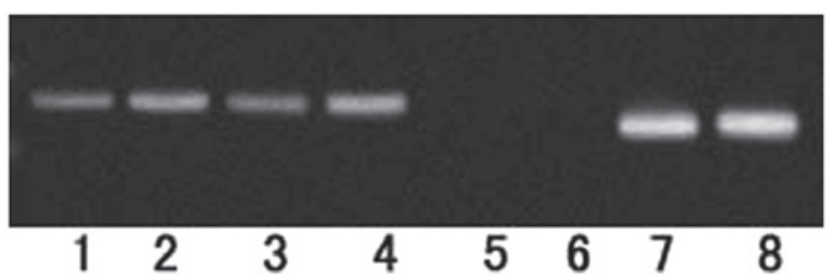

Figure 3. The Tmub1 gene promoter region contains a C/EBP $\beta$ binding site. A chromatin immunoprecipitation assay was performed using C/EBP $\beta$-specific antibodies or control immunoglobulin $\mathrm{G}$ in BRL-3A cells and the C/EBP $\beta$-Tmub1 promoter complex was amplified by polymerase chain reaction. Cells were divided into eight groups: Lanes 1 and 2, experimental groups; lanes 3 and 4, input controls; lanes 5 and 6, negative controls; lanes 7 and 8 , positive controls. The C/EBP $\beta$ overexpression vector was transfected into the cells of the groups in lanes 2,4,6 and 8. A 208-base pair fragment was detected in the experimental (lanes 1 and 2), input (lanes 3 and 4) and positive control (lanes 7 and 8) groups, which was consistent with the predicted target fragment in the promoter region of the Tmub1 gene. C/EBP $\beta$, CCAAT/enhancer-binding protein $\beta ;$ Tmubl, transmembrane and ubiquitin-like domain containing 1.

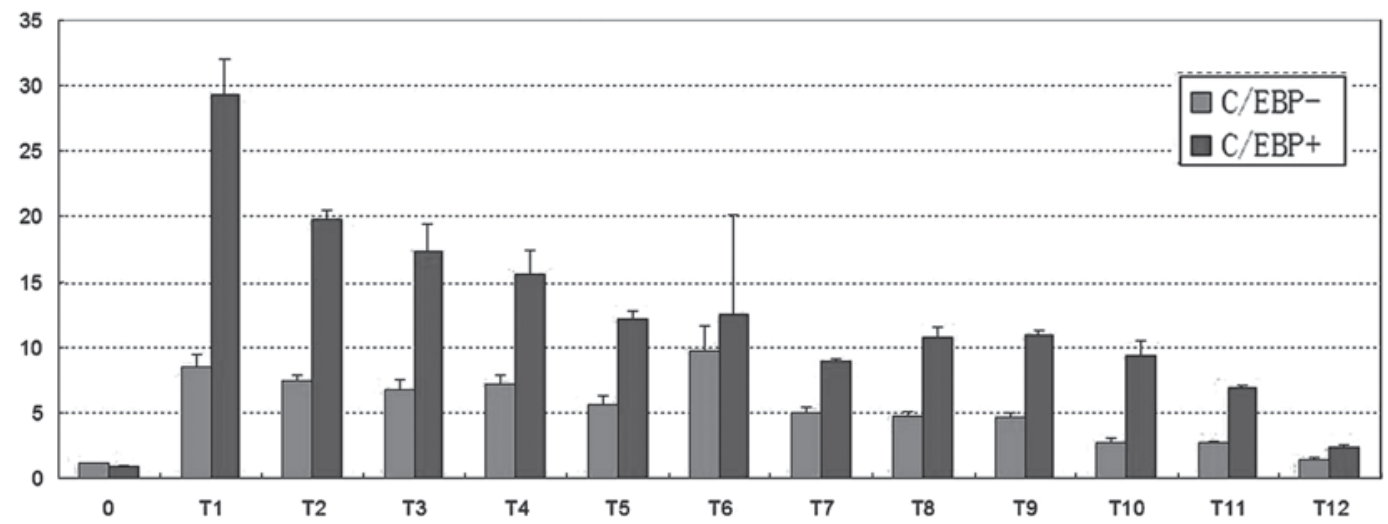

Figure 4. Effect of C/EBP $\beta$ overexpression on the activities of Tmub1 luciferase reporter vectors. The T1-T12 Tmub1 luciferase reporter vectors were co-transfected with a C/EBP $\beta$ overexpression vector or the empty pGL3-Basic vector (T0 control) into BRL-3A cells. The luciferase activities of the experimental groups were significantly higher than those of the control group $(\mathrm{P}<0.05)$. Error bars represent the mean \pm standard deviation. C/EBP $\beta$, CCAAT/enhancer-binding protein $\beta$; Tmub1, transmembrane and ubiquitin-like domain containing 1 .

the promoter activity of Tmub1 were assayed and it was identified that, during the hepatic cell proliferation process, IL-6 may enhance the expression of Tmub1 through the C/EBP $\beta$, STAT3 and AP-1 transcription factors. These findings were consistent with previous reports in the literature $(9,10)$. Among these transcription factors, STAT3 is a known characterized downstream target of IL-6, and its mechanism has been studied extensively (8). Notably, the present study showed that Tmub1 contains multiple potential $\mathrm{C} / \mathrm{EBP} \beta$ (or $\mathrm{C} / \mathrm{EBP}$ ) binding sites, and the deletion of these can significantly influence the effect of IL- 6 on enhancing the transcription of Tmub1, thus indicating that IL-6 may regulate Tmub1 expression through C/EBP $\beta$.

$\mathrm{C} / \mathrm{EBP} \beta$ is an important transcription factor required during liver regeneration and is a known downstream target of IL-6 $(13,14)$. A search of the literature and our own bioinformatics analysis have suggested that $\mathrm{C} / \mathrm{EBP} \beta$ is involved in regulating the expression of Tmub1 $(9,10,13,15,20)$, and this is supported by the data presented in this study. It was observed that inhibiting the expression of $\mathrm{C} / \mathrm{EBP} \beta$ by siRNA caused a corresponding reduction in Tmubl expression, whilst enhancing the expression of $\mathrm{C} / \mathrm{EBP} \beta$ correlated with an increase in Tmubl expression, indicating an involvement of $\mathrm{C} / \mathrm{EBP} \beta$ in the positive regulation of Tmub1 gene expression. ChIP experiments confirmed that C/EBP $\beta$ could bind with the promoter sequences of Tmub1 in proliferating liver cells, indicating that $\mathrm{C} / \mathrm{EBP} \beta$ may directly interact with Tmub1 to initiate or enhance Tmubl expression. Luciferase reporter experiments revealed that the fragment sequences in the Tmub1 promoter region (e.g. $-2,008$ to $-1,735,-1,392$ to $-1,268,-273$ to -153 and -152 to $-58 \mathrm{bp}$ ) could significantly influence the transcriptional effect mediated by $\mathrm{C} / \mathrm{EBP} \beta$, further suggesting that $\mathrm{C} / \mathrm{EBP} \beta$ binding sites may be present in these sequences. All three regions contained predicted C/EBP $\beta$ binding sites, with one binding site (-1,392 to $-1,268 \mathrm{bp})$ fragment confirmed by ChIP. The fragment sequence -152 to $-58 \mathrm{~b} b \mathrm{~b}$ had no predicted $\mathrm{C} / \mathrm{EBP} \beta$ binding site, but contained a STAT3 binding site that affected the transcriptional effect mediated by C/EBP $\beta$. Taken together, this indicated that $\mathrm{C} / \mathrm{EBP} \beta$ and STAT3 may collaboratively regulate Tmub1 transcription.

This study has shown that Tmub1 expression is regulated by IL-6, together with C/EBP $\beta$. This demonstrates that there is a close association between Tmub1, IL-6 and C/EBP $\beta$, which may play important roles in signal transduction during liver regeneration. The activation of STAT3 through the Janus kinase (JAK) pathway to induce downstream target gene expression is a known regulatory mechanism of IL-6. C/EBP $\beta$ is also considered one of the downstream targets of IL-6 and its expression can be regulated by IL- 6 during liver regeneration $(21,22)$, indicating that IL-6 can upregulate expression of $\mathrm{C} / \mathrm{EBP} \beta$ to enhance the expression of Tmub1. The results of this study support these described mechanisms reported in the literature. It is therefore hypothesized that Tmubl expression during the process of liver regeneration may be regulated by the following mechanism: IL-6-induced activation of STAT3 
via the JAK pathway and simultaneous activation of $\mathrm{C} / \mathrm{EBP} \beta$ to promote Tmub1 expression. It is also possible that $\mathrm{C} / \mathrm{EBP} \beta$ and STAT3 transcription factors act synergistically to enhance the expression of Tmub1.

In conclusion, $\mathrm{C} / \mathrm{EBP} \beta$ has been shown to be a key transcription factor involved in the regulation of Tmubl expression. During the process of liver regeneration, positive regulators, such as IL-6 and C/EBP $\beta$, can increase proliferation-related gene expression and can, at the same time, induce or enhance the expression of inhibitory factors such as Tmub1 to limit excessive cell proliferation and maintain the stability of liver regeneration.

\section{Acknowledgements}

This study was supported in part by a grant from the Natural Science Foundation of China (no. 30972895) and from the Natural Science Foundation of Chongqing (no. 2009BA5014). The authors would like to thank Medjaden Bioscience Limited for assisting in the preparation of this manuscript.

\section{References}

1. Duncan AW and Soto-Gutierrez A: Liver repopulation and regeneration: new approaches to old questions. Curr Opin Organ Transplant 18: 197-202, 2013.

2. Kurinna $S$ and Barton MC: Cascades of transcription regulation during liver regeneration. Int J Biochem Cell Biol 43: 189-197, 2011.

3. Michalopoulos GK and DeFrances MC: Liver regeneration. Science 276: 60-66, 1997.

4. Stolz DB, Mars WM, Petersen BE, Kim TH and Michalopoulos GK: Growth factor signal transduction immediately after two-thirds partial hepatectomy in the rat. Cancer Res 59: 3954-3960, 1999.

5. Fausto N: Liver regeneration. J Hepatol 32: 19-31, 2000.

6 . Kountouras J, Boura P and Lygidakis NJ: Liver regeneration after hepatectomy. Hepatogastroenterology 48: 556-562, 2001.

7. Pahlavan PS, Feldmann RE Jr, Zavos C and Kountouras J: Prometheus' challenge: molecular, cellular and systemic aspects of liver regeneration. J Surg Res 134: 238-251, 2006.

8. Streetz KL, Luedde T, Manns MP and Trautwein C: Interleukin 6 and liver regeneration. Gut 47: 309-312, 2000.
9. Schumann RR, Kirschning CJ, Unbehaun A, Aberle HP, Knope HP, Lamping N, Ulevitch RJ and Herrmann F: The lipopolysaccharide-binding protein is a secretory class 1 acute-phase protein whose gene is transcriptionally activated by APRF/STAT/3 and other cytokine-inducible nuclear proteins. Mol Cell Biol 16: 3490-3503, 1996.

10. Brown RT, Ades IZ and Nordan RP: An acute phase response factor/NF-kappa B site downstream of the junB gene that mediates responsiveness to interleukin- 6 in a murine plasmacytoma. J Biol Chem 270: 31129-31135, 1995.

11. Osada S, Yamamoto H, Nishihara T and Imagawa M: DNA binding specificity of the CCAAT/enhancer-binding protein transcription factor family. J Biol Chem 271: 3891-3896, 1996.

12. Takiguchi $\mathrm{M}$ : The $\mathrm{C} / \mathrm{EBP}$ family of transcription factors in the liver and other organs. Int J Exp Pathol 79: 369-391, 1998.

13. Greenbaum LE, Li W, Cressman DE, Peng Y, Ciliberto G, Poli V and Taub R: CCAAT enhancer- binding protein beta is required for normal hepatocyte proliferation in mice after partial hepatectomy. J Clin Invest 102: 996-1007, 1998.

14. Akira S, Isshiki H, Sugita T, Tanabe O, Kinoshita S, Nishio Y, Nakajima T, Hirano T and Kishimoto T: A nuclear factor for IL-6 expression (NF-IL6) is a member of a C/EBP family. EMBO J 9: 1897-1906, 1990

15. Akira S and Kishimoto T: IL-6 and NF-IL6 in acute-phase response and viral infection. Immunol Rev 127: 25-50, 1992.

16. Akira S: IL-6-regulated transcription factors. Int J Biochem Cell Biol 29: 1401-1418, 1997.

17. Mayer C, Gruber HJ, Landl EM, Pailer S, Scharnagl H, Truschnig-Wilders $M$ and März W: Rosuvastatin reduces interleukin-6-induced expression of C-reactive protein in human hepatocytes in a STAT3- and C/EBP-dependent fashion. Int J Clin Pharmacol Ther 45: 319-327, 2007.

18. Della Fazia MA, Castelli M, Bartoli D, Pieroni S, Pettirossi V, Piobbico D, Viola-Magni M and Servillo G: HOPS: a novel cAMP-dependent shuttling protein involved in protein synthesis regulation. J Cell Sci 118: 3185-3194, 2005.

19. Liu M, Liu H, Wang X, Chen P and Chen H: IL-6-induction of hepatocyte proliferation through the Tmub1-regulated gene pathway. Int J Mol Med 29: 1106-1112, 2012.

20. Flodby P, Antonson P, Barlow C, Blanck A, Porsch-Hällström I and Xanthopoulos KG: Differential patterns of expression of three C/EBP isoforms, HNF-1, and HNF-4 after partial hepatectomy in rats. Exp Cell Res 208: 248-256, 1993.

21. Cressmann DE, Diamond RH and Taub R: Rapid activation of the Stat3 transcription complex in liver regeneration. Hepatology 21: 1443-1449, 1995.

22. Niehof M, Streetz K, Rakemann T, Bischoff SC, Manns MP, Horn $\mathrm{F}$ and Trautwein $\mathrm{C}$ : Interleukin-6-induced tethering of STAT3 to the LAP/C/EBPbeta promoter suggests a new mechanism of transcriptional regulation by STAT3. J Biol Chem 276: 9016-9127, 2001. 